\title{
Implicancias de las cirugías estéticas en la salud física y psíquica de menores de edad
}

\section{Implications of cosmetic surgery in the physical and psychic health in underage people}

\author{
Romina Allison Bulege Núñez' \\ Universidad Peruana de Ciencias Aplicadas \\ rominabulege@hotmail.com
}

\section{RESUMEN}

El objetivo ha sido conocer en qué medida las cirugías estéticas en menores tienen implicancias en la salud física y psíquica, para ello se ha realizado la revisión de casos y experiencias en nuestro país y el extranjero. La oportunidad de hacerse una cirugía estética es muy difundida, y sobre todo se habla de los beneficios; sin embargo, lo que no hacen es ocuparse de los riesgos ni de los efectos psicológicos que en los menores de edad. De acuerdo con la Asociación Americana de Cirujanos Plásticos, por cada adolescente que se opera, 10 más están en Internet investigando el procedimiento quirúrgico que desean hacerse. Algunas conclusiones son que, la aplicación de cirugías estéticas en adolescentes se ve drásticamente afectada por la inestabilidad emocional e inmadurez propia de su etapa de desarrollo, el inconcluso crecimiento anatómico y los riesgos que toda intervención acarrea. Los países que registran mayores casos de estas cirugías están tomando medidas para proteger a menores como una acción de salud pública. Toda cirugía estética en menores exige evaluación psicológica previa. Aún queda a criterio propio la aprobación de esta estas cirugías, pero con la información preventiva correspondiente, priorizando siempre el bienestar del paciente y evitando al máximo exponerlo a cualquier tipo de riesgo físico o psicológico. Las cirugías estéticas en adolescentes no deberían estar permitidas, para ello consideré las implicaciones psicológicas como un factor determinante.

\section{ABSTRACT}

The objective was to determine the implications of cosmetic surgeries in underage people in physical and psychic health, for that the cases and experiences review in our country and abroad were made. The opportunity to have a cosmetic surgery is widespread, especially talking about the benefits; however, people don't deal with risks or psychological effects on underage people. According to the American Association of Plastic Surgeons, for each teenager that have a surgery, 10 more teens are on the Internet researching the surgical procedure they want to have. Some conclusions are that the cosmetic surgery application in teenagers is drastically affected by emotional instability and immaturity proper of their development stage, the unfinished anatomical growth and the risks that any intervention entails. The countries with the major surgeries cases are taking steps to protect children as a public health action. All cosmetic surgery on underage people requires prior psychological evaluation. It remains in our own decision to approve these surgeries, but with the appropriate precautionary information, always prioritizing the patient welfare and avoiding the exposure to any physical or psychological risk. Cosmetic surgery in teenagers shouldn't be allowed, it's important to consider the psychological implications as a determining factor.

Keywords: Cosmetic surgeries, physical health, mental health, underage people (children).

Palabras clave: Cirugias estéticas, salud física, salud psíquica, menores de edad.

Historial del artículo:

Recibido: 7 de febrero de 2015. Aprobado: 19 de mayo de 2015. Disponible en línea: 30 de junio de 2015

1 Estudiante de la Facultad de Medicina Humana de la Universidad Peruana de Ciencias Aplicadas, Lima, Perú. 


\section{INTRODUCCIÓN}

La Asociación Americana de Cirujanos Plásticos registra hasta el 2008 un total de 300000 adolescentes con al menos una intervención estética. Hoy las cifras se han incrementado en un considerable porcentaje y la recurrencia a las cirugías plásticas se extiende cada vez por más países (1). En Estados Unidos en el año 2014, 15,6 millones de procedimientos cosméticos incluyendo las mínimamente invasivas y de cirugía se llevaron a cabo, respecto al año 2013 hubo un incremento del 3 por ciento (2).

Los medios publicitarios promueven el ideal de "mujer bonita" con un prototipo de mujer delgada, cintura pequeña y grandes pechos y glúteos.
De acuerdo con la Asociación Americana de Cirujanos Plásticos, por cada adolescente que se opera, 10 más están en Internet investigando el procedimiento quirúrgico que desean (1). Para los especialistas, Charlotte hizo notar, más que su cuerpo, el alarmante incremento de cirugías injustificadas y su aplicación en adolescentes (3).

La controversia generada opone a aquellos que consideran la cirugía estética en adolescentes como un factor de riesgo y a la vez una decisión para la que no están preparados, en su mayoría afrontan episodios de crisis y falta de identidad. Estos consideran, en cambio, muy importante el grado de madurez y aceptación ante la decisión de modificar la apariencia física ¿Serán los adolescentes capaces de lidiar con algún resultado no deseado? ¿Son los adolescentes lo suficientemente maduros para decidir la modificación

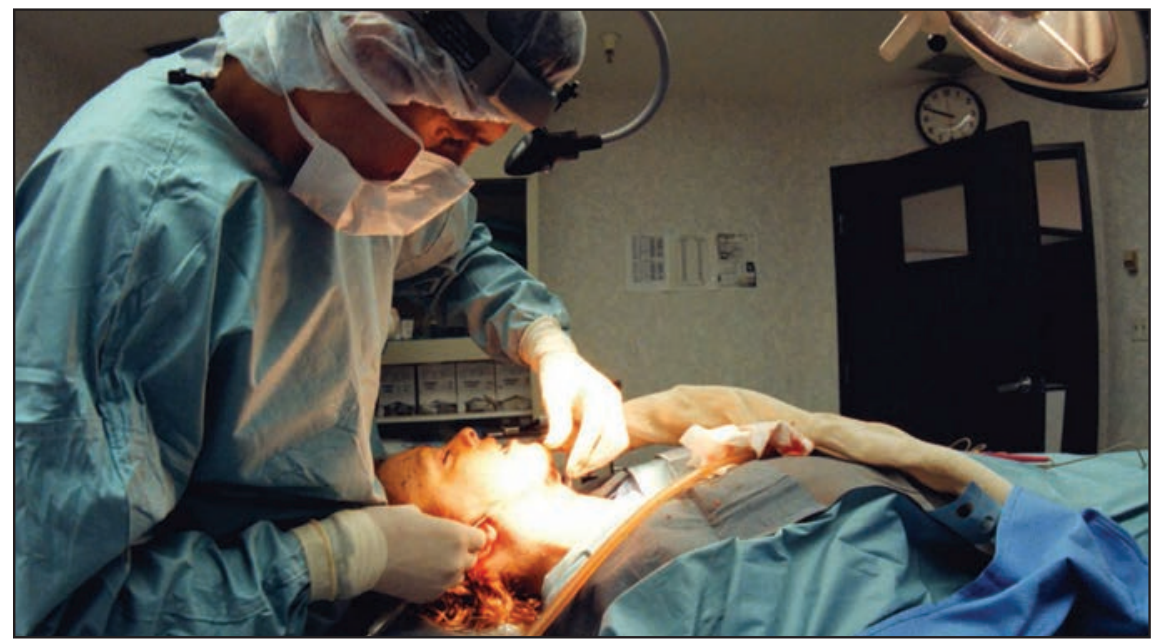

Figura $\mathrm{N}^{0} 1$ : Intervención quirúrgica en una cirugía estética.

Fuente: Zoom news. 2014. Recuperado de http://www.zoomnews.es/343849/estilo-vida /bienestar/hacerse-cirugia-estetica-sentirse-mejor

El conocido caso de Charlotte Caniggia generó polémica en Argentina y hasta llevó a que un sector de la clase política de ese país planteara la prohibición de la cirugía plástica en menores de edad como una medida de proteger la salud de los adolescentes. La hija del exfutbolista Claudio Paul Caniggia se sometió en el 2003, cuando solo tenía 20 años y ya era figura televisiva, a una múltiple cirugía estética porque simplemente su sueño era tener la imagen de la muñeca Barbie (figura $N^{\circ} 1$ ). Limadura de nariz, lipoaspiración de rodillas y cintura e implante de prótesis mamarias de $600 \mathrm{~cm} 3$ transformaron de manera radical y sorprendente su apariencia (3).

Sobre estos sucesos los diversos medios suelen desarrollar una amplia cobertura y hasta difunden los beneficios de someterse al bisturí y las respectivas facilidades; sin embargo, lo que no hacen es ocuparse de los riesgos ni de los efectos psicológicos que implica. de su apariencia por el resto de su vida? En el otro lado están quienes justifican su aplicación por la mejora anímica presenciada en varios adolescentes después de la operación, reduciendo así al mínimo las actitudes depresivas y dándoles mayor seguridad en sí mismos.

\section{Factores a evaluar previos a una cirugía estética}

Existen diversos factores a considerar previos a una cirugía estética en adolescentes y muchos de estos aspectos están directamente relacionados con su salud física o mental. Entre ellos, que la estructura anatómica a operar haya alcanzado su completo desarrollo, los especialistas desaconsejan las intervenciones en menores de 18 años porque diversas partes de su cuerpo aún no están consolidadas. En el caso de la 
rinoplastia, una de las cirugías más solicitadas por los adolescentes, el desarrollo de la nariz, por ejemplo, finaliza en la mujer a los 14 años y en el varón a los 16 años. Es importante también el estado anímico del paciente, más todavía tratándose de adolescentes cuya etapa comprende múltiples conflictos como la adquisición de nuevas responsabilidades, la vocación, los estadios de depresión o soledad, miedos, etc. Los adolescentes son asimismo, en mayor o menor grado, inmaduros emocionalmente y carecen de una identidad sólida por estar en proceso de formarla, por lo que resulta complicado definir su toma de decisiones y se pone en tela de juicio la convicción y los motivos que llevan a solicitar la intervención.

Algunos autores de la psicología como Erik Erikson en su obra Las 8 edades del hombre hablan de cómo en la pubertad y adolescencia se pone en duda todo aquello en lo que se confiaba e incluso la identidad propia. "Los adolescentes se preocupan por lo que parecen ser ante los ojos de los demás en comparación con lo que ellos mismos sienten que son" (4).

Teniendo en cuenta los presentados conflictos psicológicos que los adolescentes suelen padecer, żes viable darles la opción de cambiar drásticamente su vida, sabiendo que poco tiempo después podrían arrepentirse de ello?

Otro factor a considerar es el tener expectativas realistas de los resultados, el problema de mayor incidencia en los adolescentes son sus falsas expectativas al acudir al cirujano plástico, ellos en su mayoría esperan mejorar su autoestima y conseguir una mayor aceptación social, actitud distorsionada de la realidad porque la valoración propia no puede depender de rasgos físicos ni afectar la autoestima, entendible solo en casos de deformidad. Para encontrar el origen de este problema y tratarlo correctamente se requiere asesoría psicológica, terapias y entrenamiento en habilidades sociales. Con un adecuado autoconocimiento y aceptación podremos considerar si realmente queremos cambiar nuestra apariencia y confrontar las complicaciones si tuvieran lugar.

La mayoría de personas que optan por una intervención estética tienen una baja valoración de su imagen corporal. La "imagen de cuerpo" se define como un "concepto netamente psicoanalítico gracias a que el ser humano puede vivir como una entidad individual, diferenciada, unificada y constante" (5), la manera en que juzgamos nuestra corporalidad es el conjunto de percepciones a través de los sentidos influenciados por la idea de "belleza" que tiene la sociedad.

Una desvaloración de la imagen corporal trae como consecuencia ciertas reacciones depresivas presentes en muchos adolescentes quienes se sienten cohibidos de mostrar sus cuerpos por considerarlos inferiores. Una razón adicional para no tomar a la ligera las intervenciones estéticas en adolescentes es que en algunos casos la desvaloración de la imagen corporal puede formar parte de un trastorno psicológico y erróneamente ser operado cuando el problema debería ser tratado por un psiquiatra, el discernir si los motivos de depresión son los defectos estéticos o trastornos psiquiátricos es de gran importancia, ello ayudaría a identificar alteraciones que requieren de tratamiento, las más frecuentes son la dismorfofobia o vana creencia de tener un cuerpo feo, y la halitosis o creencia injustificada del mal olor propio, entre otras.

Entre las principales cirugías reparadoras o reconstructivas, cuyo objetivo es devolver la normalidad perdida o nunca tenida y por tanto con mayores probabilidades de solucionar algún problema de autoestima, son aceptadas la ginecomastia en varones, o la corrección de asimetrías mamarias, y la otoplastia, corrección de orejas sobresalientes.

\section{¿Cómo evaluar si un menor está apto para una cirugía estética?}

La función de los padres juega un papel importante, según la Sociedad Americana de Cirujanos Plásticos (American Society of Plastic Surgeons) deben responder a tres preguntas para evaluar qué tan aptos están sus hijos para una cirugía estética. Estas son: 1) ¿̇Es realmente el adolescente por sí mismo, el que desea la cirugía o el cambio?; 2) ¿̇es realista el adolescente acerca de los beneficios que se puede obtener con la cirugía estética o con el cambio que pretende?; y 3) ¿̇puede ser el adolescente maduro para apreciar los riesgos y desventajas de la cirugía estética? Una vez que evalúen la madurez de sus hijos, serán más prudentes al consentir o no el cambio pedido (figura $N^{\circ} 2$ ), y de encontrarse algún trastorno depresivo, estarán a tiempo para tratarlo.

\section{Prácticas de cirugia estética en el contexto internacional}

En Perú, las cirugías estéticas de mayor demanda son: de Senos (Aumento de senos con implantes, levantamiento de senos), procedimientos en Rostro y Cuello (Levantamiento de rostro, levantamiento de cuello, aplicación de Botox, aumento de labios, cirugía de parpados o blefaroplastia, aplicación de grasa, rejuvenecimiento facial), y procedimientos en otras partes del cuerpo (6). Si bien las cifras en relación con la sobredemanda de cirugías estéticas en adolescentes en nuestro país no es preocupante, no debemos esperar que suceda para actuar al respecto.

Países como Argentina, España y Estados Unidos, cuyos casos presentaremos, han implementado mecanismos 
de restricción para de alguna manera reducir esta tendencia de la moda que está marcando a su población joven. Una cirugía en estos países puede ser el regalo de cumpleaños de una quinceañera o simplemente un gusto más que pagar. Las personas y médicos tomaron a la ligera las implicancias de ser adolescente.

En Argentina la diputada del partido gobernante, Mara Brawer, lleva a cabo un proyecto de ley que prohíbe las cirugías estéticas en menores de 18 años, con el objetivo de preservar la salud física y psíquica de este sector de la población. Brawer, también psicóloga de profesión, propone en su proyecto multas de hasta US\$ 625000 y la inhabilitación temporaria de la matrícula, según la gravedad del caso, a los médicos que intervengan a menores de 18 años. La iniciativa exceptúa de la prohibición las intervenciones reconstructivas, reparadoras, terapéuticas 0 de intervención, el ideal es descartar posibles trastornos psicológicos, por lo que todo menor de 18 años que quiera someterse a una cirugía estética deberá realizar un examen psicológico previo.

El decreto establece también los requisitos que deben cumplir los centros y profesionales a cargo de las intervenciones. Con este objetivo se elaboraron diversos documentos tanto para el personal sanitario como para los ciudadanos. Para el personal sanitario, entre otros documentos, fueron trabajadas guías de uso clínico y asistencial, consideraciones e implicaciones psicológicas, consentimiento informado que tiene que firmar el paciente; para los ciudadanos, en tanto, una serie de documentos en los que se informarán sobre las principales implicaciones de las cirugías estéticas más demandadas (7).

En Estados Unidos también se busca regular la

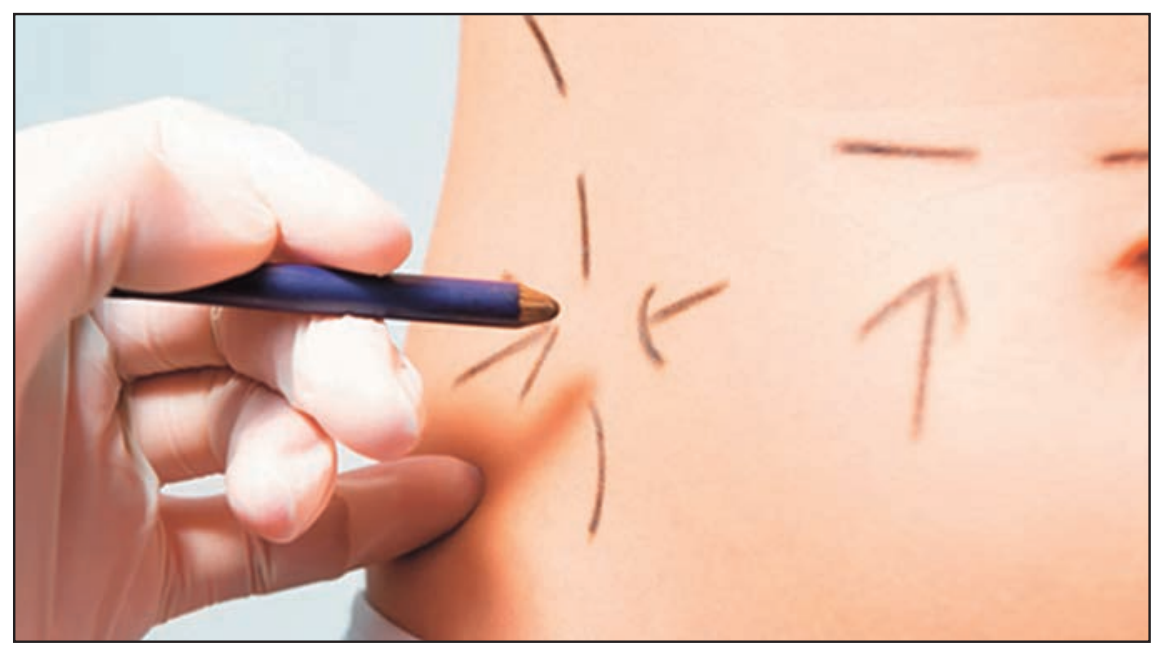

Figura $\mathrm{N}^{\circ}$ 2: Cirugías estéticas en adolescentes deben hacerse previa evaluación.

Fuente: Roch, clínica de medicina estética. 2014. Recuperado de: http://www.clinicaroch. com/cirugia_estetica_genital_sevilla.php

adecuación del cuerpo a la identidad de género, y en caso los rasgos generen estigmatizaciones, queda permitida la intervención pero previo informe médico (3).

En España, uno de los países con mayor demanda juvenil de cirugías plásticas, se estima un promedio de 380000 operaciones anuales y el incremento de esta tasa entre un $5 \%$ y $10 \%$, ritmo que sitúa a dicho país en cuarto lugar en el ámbito mundial en el rubro.

Estadísticamente una de cada diez intervenciones se realiza a menores de edad, por ello la Junta de Andalucía ha desarrollado un decreto que norma la práctica de estas intervenciones priorizando la seguridad de los menores. El decreto limita las operaciones a casos estrictamente documentados donde se justifique los riesgos y beneficios de la cantidad y los efectos de la cirugía plástica en adolescentes, los especialistas recomiendan esperar la adultez para realizarse las intervenciones requeridas y han impuesto límites de edad y obtención de recursos como no aprobar el uso de implantes mamarios en menores de 22 años. Otra iniciativa es la promoción de hábitos saludables en la rutina diaria en reemplazo de la lipoaspiración, operación que conlleva muchos riesgos y que en su mayoría podrían evitarse.

Colombia, el sexto país donde se hacen más procedimientos, según datos de la Sociedad Internacional de Cirugía Plástica Estética, se realizaron 420955 procedimientos estéticos en 2013, lo que ubica al país en el sexto lugar en el mundo con mayor número de cirugías, después de Estados Unidos, Brasil, México, Alemania y España (8). 


\section{CONCLUSIONES}

Ciertos profesionales consideran que la cirugía estética en adolescentes es factible porque se ha demostrado su efecto en miles de personas que efectivamente mejoraron su autoestima y problemas de inseguridad. Como mencionamos, cuando las cirugías son para restablecer la normalidad en caso de accidentes o deformaciones, existen altas probabilidades de obtener resultados positivos pues porque en estos casos se está solucionando el problema desde su origen, sin embargo la mayoría de adolescentes busca operarse con el fin de conseguir mayor aceptación social, un claro problema de inseguridad, y ello no puede ser resuelto por una cirugía, las inseguridades no desaparecen, en cuanto se le opere de alguna parte, posiblemente busque otro defecto y seguirá así hasta dar una solución al origen que en estos casos requieren terapia psicológica.

La aplicación de cirugías estéticas en adolescentes se ve drásticamente afectada por la inestabilidad emocional e inmadurez propia de su etapa de desarrollo, el inconcluso crecimiento anatómico y los riesgos que toda intervención acarrea. Los países que registran mayores casos de estas cirugías en adolescentes están tomando medidas para proteger a este sector población como una acción de salud pública. Toda cirugía estética en menores de edad exige evaluación psicológica previa y que en casos de cirugía reconstructiva existen menos restricciones porque solo busca devolver la normalidad. Aún queda a criterio propio la aprobación de esta estas cirugías, pero con la información preventiva correspondiente, priorizando siempre el bienestar del paciente y evitando al máximo exponerlo a cualquier tipo de riesgo físico o psicológico.

Las cirugías estéticas en adolescentes no deberían estar permitidas, para ello consideré las implicaciones psicológicas como un factor determinante.

\section{REFERENCIAS BIBLIOGRÁFICAS}

1. Cirugías estéticas adolescentes żnecesidad o vanidad? [Internet]. San Diego: Tania Luviano; 25 de agosto de 2011 [citado 14 de enero de 2015]. Vida y estilo [1 pantalla]. Disponible en: http://vidayestilo.terra.com/cirugias-esteticasadolescentes-necesidad-o-vanidad, 12clafcb 192 02310VgnVCM20000099f154d0RCRD.html

2. Plastic Surgery Procedural Statistics [Internet]. Arlington: American Society of Plastic Surgeons; 28 de diciembre de 2014 [citado 14 de enero de 2015]. News [1 pantalla]. Disponible en: http:// www. plasticsurgery.org/news/plastic-surgerystatistics.html

3. ¿ ¿Se debe prohibir la cirugía estética a los menores de edad? [Internet]. Buenos Aires: BBC Mundo; 30 de mayo de 2014 [citado 17 de diciembre de 2014]. Salud [1 pantalla]. Disponible en: http://www. bbc.co.uk/mundo/noticias/2014/05/140528_ argentina_cirugia_estetica_menores_vs

4. Bordignon NA. El desarrollo psicosocial de Eric Erikson - El diagrama epigenético del adulto. Revista Lasallista de investigación [Internet]. Febrero de 2006 [citado 17 de diciembre de 2014]; 2(2): 4-58. Disponible en: http://www. lasallista.edu.co/fxcul/media/pdf/Revista/vol2n2/ p50-63 ARTICULO\%20ERICK\%20ERICKSON.pdf

5. Guimón J. Cirugía estética: implicaciones psicológicas. ASMR Revista Internacional On-Line [Internet]. 2008 Agosto [Citado el 17 de diciembre de 2014]; 7(3): [1 pantalla]. Disponible en: http:// www.psiquiatria.com/revistas/index.php/asmr/ article/view/52/

6. Procedimientos solicitados en cirugía estética [Internet]. Lima: Cirugia estética en Perú; 14 de julio de 2013 [citado 11 de diciembre de 2014]. Procedimientos mas solicitados en cirugía estética [1 pantalla]. Disponible en: http:// www.sociedadcirugiaplasticaperu.com/estetica/ procedimientos-solicitados-de-cirugia-estetica. $\mathrm{htm}$

7. Cirugía estética en menores [Internet]. Sevilla: Consejería de Igualdad, Salud y Políticas Sociales, Junta de Andalucia; 23 de octubre 2012 [citado 12 de enero de 2015]. Nuestra salud [1 pantalla]. Disponible en: http://www.juntadeandalucia. es/salud/sites/csalud/contenidos/Informacion General/c_3_c_1_vida_sana/adolescencia/ decreto_cirugia_estetica_menoress_vs

8. Cirugía plástica en menores ìrecomendable? [Internet]. Barranquilla: miercoles; 15 de abril de 2015 [citado 22 de abril de 2015]. Actualidad [1 pantalla]. Disponible en: http://revistas.elheraldo. $\mathrm{co} / \mathrm{miercoles} /$ actualidad/cirugia-plastica-enmenores-recomendable-133672 